\title{
Rethinking the thinking cap
}

\section{Ethics of neural enhancement using noninvasive brain stimulation}

Roy Hamilton, MD, MS

Samuel Messing, BA

Anjan Chatterjee, MD

Address correspondence and reprint requests to Dr. Roy H. Hamilton, 3 Gates Building, Hospital of the University of Pennsylvania, 3400 Spruce Street, Philadelphia, PA 19104 royhhamilton@yahoo.com

\section{ABSTRACT}

Although a growing body of evidence suggests that noninvasive brain stimulation techniques such as transcranial magnetic stimulation and transcranial direct current stimulation have the capacity to enhance neural function in both brain-injured and neurally intact individuals, the implications of their potential use for cosmetic self-enhancement have not been fully explored. We review 3 areas in which noninvasive brain stimulation has the potential to enhance neurologic function: cognitive skills, mood, and social cognition. We then characterize the ethical problems that affect the practice of cosmetic neurology, including safety, character, justice, and autonomy, and discuss how these problems may apply to the use of noninvasive brain stimulation for selfenhancement. Neurology ${ }^{\circledR}$ 2011;76:187-193

\section{GLOSSARY}

DLPFC = dorsolateral prefrontal cortex; LATL = left anterior temporal lobe; $R$ RA $=$ remote association test; $\mathbf{r T M S}=$ repetitive TMS; tDCS = transcranial direct current stimulation; TMS = transcranial magnetic stimulation.

THE THINKING CAP Recent years have witnessed a surge of interest in the potential for therapies developed to help patients with cognitive deficits to enhance the abilities of individuals with normal cognition. While this emerging field of cosmetic neurology offers the promise of changing how we think and feel in ways that will make us more effective in the workplace, more attentive in school, or happier in our personal lives, it has also fueled serious ethical concerns. ${ }^{1,2}$

Noninvasive brain stimulation techniques such as transcranial magnetic stimulation (TMS) and transcranial direct current stimulation (tDCS) are popular investigative tools in cognitive neuroscience, and are increasingly being explored as treatments for a variety of neurologic and psychiatric conditions (figure). Mounting experimental evidence also suggests that focal brain stimulation can transiently improve performance on a variety of cognitive tasks in otherwise healthy individuals, raising the possibility that these technologies may someday be employed cosmetically.

The notion of magnetic or electrical devices that noninvasively enhance brain activity calls to mind the proverbial thinking cap, an idea that has already captured the public imagination. ${ }^{3,4}$ In this article, we survey current work involving TMS and tDCS that relates to neurologic enhancement, reflect on ethically relevant similarities and differences between pharmacologic enhancement and noninvasive brain stimulation, and consider some potentially problematic issues that may arise with respect to certain ethical principles: nonmalfeasance and safety, justice, character and personhood, and autonomy.

OVERVIEW OF TMS AND TDCS TMS involves the generation of a rapid time-varying magnetic field in a coil of wire. When this coil is held to the head of a subject, the magnetic field penetrates the scalp and skull inducing a small current parallel to the plane of the coil in the brain that is sufficient to depolarize neuronal membranes and generate action potentials. Repetitive TMS (rTMS), which involves the application of a series of pulses at a predetermined frequency, is particularly relevant to neural enhancement because it can produce effects that outlast the application of the stimulation. Evidence suggests that rTMS delivered at a low frequency $(0.5-2 \mathrm{~Hz})$ tends to focally decrease cortical excitability, whereas higher frequencies (faster than $5 \mathrm{~Hz}$ ) tend to increase excitability. ${ }^{5}$

tDCS involves the application of small electrical currents to the scalp through 2 surface electrodes. Current flows from the anode, through the cortex, and out through the cathode. Unlike TMS, which induces currents

From the Department of Neurology (R.H., A.C.), Center for Cognitive Neuroscience (R.H., S.M., A.C.), and Laboratory for Cognition and Neural Stimulation (R.H., S.M.), University of Pennsylvania, Philadelphia.

Disclosure: Author disclosures are provided at the end of the article. 


\section{Figure Number of transcranial magnetic stimulation (TMS) and transcranial direct current stimulation (tDCS) articles published per year (1999-2009)}

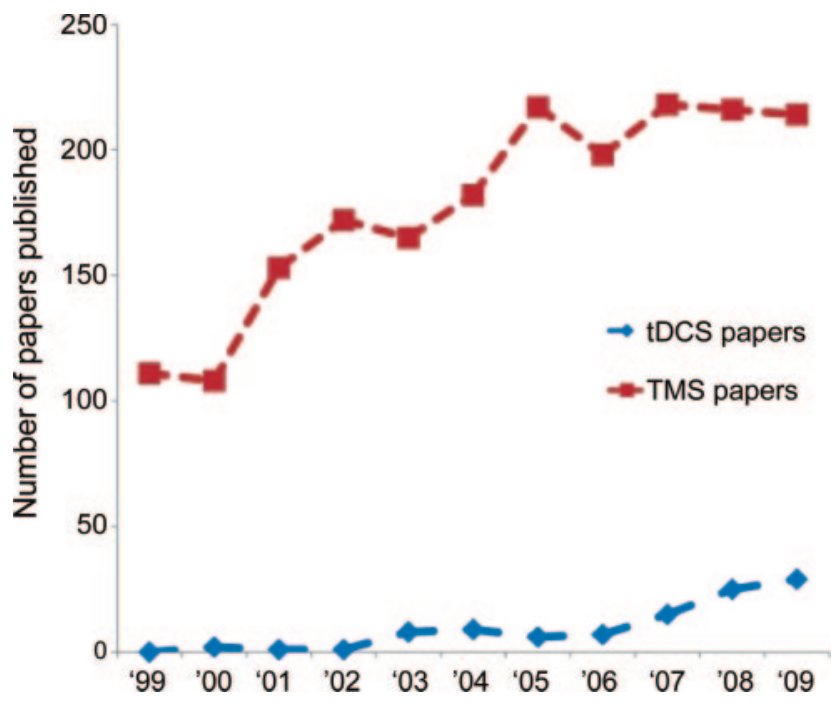

Data were acquired by searching pubmed.gov for articles created each year with "transcranial magnetic stimulation," "TMS," "transcranial direct current stimulation," or "tDCS" in the title. Review articles were excluded. All searches were conducted on March 8, 2010.

of sufficient magnitude to stimulate action potentials, the weak electrical currents employed in tDCS are thought to modulate the resting membrane potentials of neurons. The effect of tDCS depends on which electrode is applied to the scalp: cathodal stimulation is associated with decreased cortical excitability due to hyperpolarization of cortical neurons, while anodal stimulation is associated with increased cortical excitability due to subthreshold depolarization. These effects may last from minutes to hours depending on the intensity, polarity, and duration of stimulation. ${ }^{6,7}$

Comparing the 2 techniques, TMS affords greater spatial and temporal resolution than $\mathrm{tDCS}$. These differences in precision may lend themselves to different applications for TMS and tDCS depending on whether manipulation of a particular cognitive operation is more readily accomplished by focused or distributed stimulation. Also, compared to TMS, tDCS is currently less expensive, much more portable, very well-tolerated, and associated with fewer safety concerns. ${ }^{8}$

THE PROMISE OF NONINVASIVE BRAIN STIMULATION Based on studies that involve healthy individuals, noninvasive brain stimulation may eventually be used for self-enhancement in at least 3 realms: cognitive skills, mood, and social cognition.

Cognitive skills. TMS and tDCS have been used to induce transiently improved performance in healthy individuals on tasks that rely on different aspects of learning and memory. Several investigators have used noninvasive brain stimulation to affect the ability to hold and manipulate information over short periods of time, commonly known as working memory.9,10 Numerous other studies have shown that manipulation of activity in the primary motor region of cortex (M1) can improve performance on various complex motor learning tasks. ${ }^{11-13}$

Another area where the potential of cognitive enhancement holds great promise is language learning. Anodal tDCS over Broca's area has been shown to improve the ability of subjects to learn new object names and may also enhance the ability to learn novel grammar. ${ }^{14,15}$ In other investigations, TMS and tDCS of language-related regions of the left hemisphere has been shown to induce faster object naming, ${ }^{16,17}$ and anodal tDCS applied to the left prefrontal cortex has been shown to transiently improve verbal fluency. ${ }^{18}$

The evidence for enhancement of more general complex problem-solving abilities via noninvasive brain stimulation is limited, but intriguing. In one recent investigation, tDCS of the left dorsolateral prefrontal cortex (DLPFC) improved performance on the remote association test (RAT), a task in which subjects are presented with sets of 3 words and asked to identify the common linguistic association between them (e.g., the words "scan," "wash," and "child" can be linked to the common word "brain"). ${ }^{19}$ Investigators have linked performance on the RAT task to other measures of creative thinking, executive function, and general intelligence, suggesting that noninvasive brain stimulation could potentially enhance these cognitive abilities as well.

Limited evidence also suggests that TMS and tDCS can potentially enhance visuospatial processing. In one study, low-frequency rTMS applied to the parietal lobe increased accuracy on a visual stimulus detection task for visual targets on the side that was stimulated. ${ }^{20}$ Importantly, subjects also showed a trend toward worsened detection of targets in the opposite field of vision. This latter finding suggests that the potential for noninvasive brain stimulation to increase performance on a particular cognitive task may sometimes be achieved at the expense of other cognitive operations that make use of similar brain areas, an issue we revisit in the discussion of the risks of brain stimulation.

Finally, one set of cognitive skills in which the role of TMS and tDCS has been especially controversial is the unmasking of so-called savant-like abilities. Snyder ${ }^{21}$ argued that inhibition of the left anterior temporal lobe (LATL) with brain stimulation interferes with semantic networks that normally impose top-down constraints on human perception-in short, making conception play a dimin- 
ished role in perception. These investigators suggest that suppressing the LATL increases access to lessprocessed information, improving performance in perceptual abilities on tasks such as drawing, proofreading, numerosity judgment, ${ }^{3}$ and other cognitive processes in which conceptual knowledge biases performance. ${ }^{22,23}$

Mood modulation. The use of TMS to treat depression has been reported in dozens of randomized controlled trials spanning more than a decade. ${ }^{24-26}$ This body of evidence indicates that rTMS of the prefrontal cortex leads to symptomatic improvement in some patients who have failed to respond to antidepressant medications, and in 2008 the US Food and Drug Administration approved the use of TMS for this indication. Dozens of clinical treatment centers now offer therapeutic brain stimulation (e.g., http:// www.neurostartms.com). Evidence for the efficacy of tDCS in treating depression is more preliminary but also promising. ${ }^{27,28}$

The approach used to stimulate patients with depression is based on the notion of a hemispheric imbalance of cortical activity in the prefrontal cortex, with relatively decreased activity on the left in depressed patients. $^{29,30}$ Therapeutic brain stimulation for depression has therefore generally entailed either excitation of the left or inhibition of the right prefrontal cortex. ${ }^{26}$ Unilateral stimulation of the prefrontal cortex has been shown to induce transient changes in mood in healthy individuals as well. ${ }^{31,32}$ Interestingly, for reasons that remain unclear, the mood changes induced by high-frequency rTMS in healthy persons appear to show a pattern opposite that seen in depressed patients. Elevations of mood are associated with right-sided excitation and depression of mood with left-sided excitation. These mood effects in healthy subjects have not yet been replicated using tDCS. ${ }^{33}$

The use of noninvasive brain stimulation to modulate mood raises the question of whether and how noninvasive brain stimulation may be combined with existing pharmacologic agents such as antidepressants to induce changes in affect, cognition, and personality. Several studies using tDCS have shown that agents that modulate the function of a variety of neurotransmitter systems can have a profound impact on the ability of noninvasive brain stimulation to induce excitability changes in the cortex. ${ }^{34,35}$ One can envision future investigations and treatment paradigms in which noninvasive brain stimulation is used in conjunction with pharmacologic agents.

Social cognition. Evidence suggests that TMS and tDCS can transiently alter an individual's understanding of and relationship to others in ways that touch directly on the neural basis of ethical and moral thought and behavior. For instance, Young and colleagues ${ }^{36}$ recently tested subjects on a task in which they made judgments about the moral permissibility of the intentions and actions of characters in short vignettes. TMS applied to the right temporoparietal junction caused subjects to focus more on the outcome of the act than the intention of the actor when judging permissibility of the act. This was the case even when the agent's intent was to harm another person, so long as the outcome of the agent's actions was innocuous.

In another striking example of how brain stimulation can affect social cognition, Knoch and colleagues ${ }^{37}$ employed an "ultimatum game" in which subjects were asked to either accept or refuse a share of money that was divided and offered to them by a second individual (the proposer). If the subject refused the money, neither the subject nor the proposer received any. Although it was in the selfinterest of the subject to accept any offer, subjects typically refused offers that they perceived as unfairly low. This decision is thought to rely on notions of fairness, equity, and reciprocity and is termed "altruistic punishment." After inhibitory noninvasive brain stimulation of the right DLPFC, subjects were more likely to accept low offers, even though they still perceived them as being unfair. It seems as though subjects were more motivated to act in their self-interest than to abstain from a reward for the sake of fairness. TMS and tDCS of the DLPFC have also been shown to manipulate behavior on a variety of other rewardseeking tasks. ${ }^{38}$

A third example comes from investigations into the neural basis of duplicity. Prior evidence suggests that TMS, under the right circumstances, can differentiate neural activity elicited during deceptive behavior from that elicited during honest behavior. Investigators measured the motor response generated by magnetic stimulation of the motor cortex (motor evoked potential), and found that measurements of this neurophysiologic marker during deception were larger than those generated during truthfulness. ${ }^{39}$ Moreover, Priori and colleagues ${ }^{40}$ noted that bilateral anodal tDCS delivered to the DLPFC slows the generation of deceptive responses but not truthful ones, suggesting that manipulating cortical activity in this region interferes with the capacity for deception. In addition, Luber and colleagues ${ }^{41}$ report that pulses of TMS delivered to the midline parietal cortex also selectively slows deceptive responses.

THE PERILS OF NONINVASIVE BRAIN STIMULATION It has been argued by some that the benefits of self-enhancement may outweigh social risks and that the social cost of foregoing enhancement may be con- 
siderable. ${ }^{42}$ Nonetheless, as the cosmetic use of pharmacologic neural enhancement continues to increase, it is incumbent on science and society to address several concerns: 1) safety, 2) character, 3) justice, and 4) autonomy. These same concerns are likely to be important if self-enhancement with noninvasive brain stimulation becomes more widespread.

Safety. There are known and possibly unknown safety concerns associated with noninvasive brain stimulation. The most important safety risk associated with TMS is the risk of inducing seizures. Since the introduction of published safety parameters for the use of rTMS, the incidence of induced seizure is exceptionally rare. ${ }^{43}$ All current evidence indicates that tDCS is extremely safe; its main safety risks are mild headache and a mild burning or itching sensation under the electrodes. ${ }^{44}$ Given the newness of these methodologies, one caveat is that not much is known about the chronic effects of either magnetic or electrical brain stimulation. Future studies may reveal unsuspected side effects and risks that are more serious than those associated with some conventional methods of behavioral cognitive manipulation (e.g., psychotherapy).

One potential concern that may be more relevant to noninvasive brain stimulation than to psychopharmacology is that focal brain stimulation performed to enhance some abilities may be deleterious to others. ${ }^{20,45}$ Excitation or inhibition of a brain region in order to affect one mental operation may have unintended effects on other functions, either because the region of brain stimulated is part of the integral networks of those functions or because direct manipulation of that brain region results in indirect inhibition or excitation of critical downstream neural elements. Focal manipulation of cortical function with noninvasive brain stimulation has been compared to adjusting the weights on a complicated mobile; "pushing" on one piece may have inadvertent effects on the others. ${ }^{46}$

While the safety issues associated with brain stimulation are of paramount importance, they do not comprise an especially complicated ethical dilemma. Here there is a strong analogy to be made between noninvasive brain stimulation and pharmacologic agents. As with pharmacologic agents, all parties that would be involved in the use of noninvasive stimulation for selfenhancement-patients/consumers, device producers, and practitioners - are likely to desire a safe outcome. Therefore there is no inherent conflict of interest. As such, as long as information about adverse side effects is not suppressed, safety problems seem to be of a more pragmatic than deeply ethical nature. ${ }^{2}$

Character. The issue of character relates to our notions of what defines our identity and gives meaning to our lives. Here there are at least 2 issues. The first is whether we believe fundamental elements of selfidentity can and should be altered. The second is predicting the unintended long-term consequences of self-enhancement, especially with respect to characterological and psychosocial development.

Evidence suggests that most people believe it is acceptable to change certain mental abilities, such as concentration or memory, while other qualities - for example, honesty or fairness - are considered so integral to an individual's self-identity that many people find it inappropriate to manipulate them. ${ }^{47}$ If these core qualities can be changed in an enduring way using brain stimulation, some would argue that an individual who has changed them has changed his or her identity - that he or she is now in fact a different person. A related concern is that the ability to enhance our essential traits and abilities runs the risk of commodifying them, ${ }^{48}$ and possibly of eroding our collective appreciation of human gifts, talents, and achievements. ${ }^{49}$ If the use of noninvasive brain stimulation for self-enhancement becomes widespread, it is likely that society will be forced to confront essential questions of self-identity and whether there are or should be limits on our ability to change our fundamental nature.

A separate issue is whether widespread and frequent cosmetic use of neural enhancements, especially mood enhancements, could eventually affect the development of personality traits in unintended ways. Brain stimulation and pharmacologic enhancements might someday be used electively to mitigate relatively minor negative mood states in psychiatrically healthy individuals. On one level, this seems appealing, since all of us generally seek to avoid suffering. However, the ability to endure rather than avoid physical and psychological discomfort may play a fundamental role in psychological development, in part by engendering socially valued attributes, such as patience and determination. ${ }^{50}$ Recent neuroimaging evidence even suggests that observing pain in others elicits patterns of neural activation similar to those engaged when one experiences pain directly, suggesting that painful experiences may play a role in empathy. ${ }^{51}$ Moreover, some have argued that the ability to overcome hardship may contribute to psychological wellbeing by giving rise to a sense of accomplishment and satisfaction in one's actions. ${ }^{48,52}$ Thus, while neural enhancements might someday offer the possibility of smoothing out emotional rough patches in the lives of otherwise healthy individuals, it is unclear whether this kind of manipulation will come at the expense of other valuable traits. 
Justice. This relates to the equitable distribution of resources. Like medications that can be used for cognitive enhancement in normal individuals, noninvasive brain stimulation for self-enhancement may not be covered by insurance companies. This situation may be conducive to the emergence of a small minority of individuals who are able to pay out-of-pocket for personalized boutique enhancement regimens to improve their affect and cognition. Extending this hypothetical scenario further, if cognitive enhancement becomes commonplace for a certain set of people, the range of expectations regarding what able individuals are capable of achieving may eventually shift to accommodate the augmented abilities of the "enhanced" class. Eventually, cognitive abilities may become increasingly "medicalized," to the point that unenhanced cognition may be viewed as a pathologic state by those who can afford to enhance themselves. ${ }^{53}$

While the issue of distributive justice is clearly problematic when considering the issue of cognitive enhancement, it is by no means unique to brain stimulation. Rather it would mirror the widening gap between rich and poor with respect to almost all aspects of life, including nutrition, personal safety, medical care, and education. It would amount to another reflection of a social system where, at least in the United States, these disparities have historically been tolerated.

Autonomy. With respect to autonomy, the question is not whether the ability to avail oneself of salutary brain stimulation could be curtailed (that is an issue of justice, as noted above), but rather whether situations could someday arise in which one could be explicitly or implicitly coerced to undergo brain stimulation.

Explicit coercion in this context refers to forcing individuals to undergo noninvasive brain stimulation against their will for the perceived greater good of society. Here again comparison to pharmacologic treatments is instructive. A number of US states have experimented with the concept of "chemically castrating" repeat sex offenders using medroxyprogesterone, a hormonal treatment that decreases sexual drive and aggressiveness. ${ }^{54}$ Laws such as these set a precedent for the use of coercive interventions to modify behavior and cognition in certain populations. One context in which explicit coercion can be envisioned is the detection and manipulation of deception, wherein noninvasive brain stimulation could someday add to or replace the polygraph, or be used as a neurophysiologic "truth serum" that interferes with the ability to lie. ${ }^{39-41}$ Such potential applications of brain stimulation would call into question whether the state, under any circumstances, has the right to elucidate or manipulate cognitive states against one's will.

Implicit coercion in this context refers to the pressure to engage in brain enhancement as a way to keep up with the demands of a competitive society. Progress today is widely considered to involve the unrelenting pursuit of greater efficiency and productivity. For many individuals intense competition and the drive to succeed characterizes life in the workplace, school, and even at home. In a society where pharmacologic doping frequently mars the fairness of professional sports and where both students and their professors take stimulant medications to meet their academic expectations, ${ }^{55,56}$ the potential pressure for the use of cognitive enhancing technologies of all types is very real.

DISCUSSION We have tried to make the case that the use of noninvasive brain stimulation for selfenhancement is not far-fetched. While we do not assert that elective brain stimulation is inevitable, the emerging popularity of other forms of cosmetic brain enhancement implies that noninvasive brain stimulation may also someday become part of the mainstream. We have also emphasized the ethical issues that are likely to arise if the cosmetic use of noninvasive brain stimulation becomes more widespread, in particular those related to character, justice, and autonomy.

Cosmetic applications of noninvasive brain stimulation may challenge the traditional role of physicians, especially neurologists and psychiatrists, who are currently the principal clinical practitioners of TMS and tDCS. Plastic surgeons faced a similar challenge to their identity in the last century as the demand for their services shifted from reconstructive to cosmetic procedures, and clinical neurosciences currently face a similar challenge with respect to pharmacologic cognitive enhancement. ${ }^{57}$ Physicians will need to clarify their relationships with patients and consumers, especially when their fiduciary and financial interests are at odds. Moreover, as novel approaches for enhancing cognition develop, accurate dissemination of information from the scientific and medical world to the public will call for the responsible actions of clinicians and neuroscientists alike. Physicians and neuroscientists will also need to partner to ensure that standards of safe and appropriate usage can be implemented as technologies like noninvasive brain stimulation transfer from the laboratory to the clinic to cosmetic use. ${ }^{58}$

\section{DISCLOSURE}

Dr. Hamilton receives research support from the NIH/NINDS (1K01NS060995 [PI]) and the Robert Wood Johnson Foundation/ Harold Amos Faculty Development Program. S. Messing holds stock in Medical Information Technology Inc. Dr. Chatterjee serves as an Associ- 
ate Editor for the Journal of Cognitive Neuroscience and receives research support from the NIH (RO1 DC004817 [PI] and RO1 DC008779 [PI]) and the National Science Foundation.

Received May 26, 2010. Accepted in final form August 30, 2010.

\section{REFERENCES}

1. Chatterjee A. Cosmetic neurology: the controversy over enhancing movement, mentation and mood. Neurology 2004;63:968-974.

2. Chatterjee A. The promise and predicament of cosmetic neurology. J Med Ethics 2006;32:110-113.

3. Snyder AW, Mulcahy E, Taylor JL, Mitchell DJ, Sachdev P, Gandevia SC. Savant-like skills exposed in normal people by suppressing the left fronto-temporal lobe. J Integr Neurosci 2003;2:149-158.

4. Hanuain D. Thinking cap or dunce's hat? Wired April 18, 2002.

5. Maeda F, Pascual-Leone A. Transcranial magnetic stimulation: studying motor neurophysiology of psychiatric disorders. Psychopharmacology 2003;168:359-376.

6. Priori A. Brain polarization in humans: a reappraisal of an old tool for prolonged non-invasive modulation of brain excitability. Clin Neurophysiol 2003;114:589-595.

7. Nistche MA, Paulus W. Excitability changes induced in the human motor cortex by weak transcranial direct current stimulation. J Physiol 2000;572:633-639.

8. George MS, Aston-Jones G. Noninvasive techniques for probing neurocircuitry and treating illness: vagus nerve stimulation (VNS), transcranial magnetic stimulation (TMS) and transcranial direct current stimulation (tDCS). Neuropsychopharmacology 2010;35:301-316.

9. Ohn SH, Park CI, Yoo WK. Time-dependent effect of transcranial direct current stimulation on the enhancement of working memory. Neuroreport 2008;19:43-47.

10. Fregni F, Boggio PS, Nitsche M. Anodal transcranial direct current stimulation of prefrontal cortex enhances working memory. Exp Brain Res 2005;166:23-30.

11. Kim YH, Park JW, Ko MH, Jang SH, Lee PK. Facilitative effect of high frequency subthreshold repetitive transcranial magnetic stimulation on complex sequential motor learning in humans. Neurosci Lett 2004;367:181-185.

12. Nitsche MA, Schauenburg A, Lang N, et al. Facilitation of implicit motor learning by weak transcranial direct current stimulation of the primary motor cortex in the human. J Cogn Neurosci 2003;15:619-626.

13. Kobayashi M, Hutchinson S, Théoret H, Schlaug G, Pascual-Leone A. Repetitive TMS of the motor cortex improves ipsilateral sequential simple finger movements. Neurology 2004;62:91-98.

14. Flöel A, Rösser N, Michka O, Knecht S, Breitenstein C. Noninvasive brain stimulation improves language learning. J Cogn Neurosci 2008;20:1415-1422.

15. de Vries MH, Barth AC, Maiworm S, Knecht S, Zwisterlood P, Flöel A. Electrical stimulation of Broca's area enhances implicit learning of an artificial grammar. J Cogn Neurosci Epub 2009 Nov 19.

16. Mottaghy FM, Hungs M, Brügmann M, et al. Facilitation of picture naming after repetitive transcranial magnetic stimulation. Neurology 1999;53:1806-1812.

17. Sparing R, Dafotakis M, Meister IG, Thirugnanasambandam N, Fink GR. Enhancing language performance with non-invasive brain stimulation: a transcranial direct cur- rent stimulation study in healthy humans. Neuropsychologia 2008;46:261-268.

18. Iyer MB, Mattu U, Grafman J, Lomarev M, Sato S, Wassermann EM. Safety and cognitive effect of frontal DC brain polarization in healthy individuals. Neurology 2005; 64:872-875.

19. Cerruti C, Schlaug G. Anodal transcranial direct current stimulation of the prefrontal cortex enhances complex verbal associative thought. J Cogn Neurosci 2009;21:19801987.

20. Hilgetag CC, Théoret H, Pascual-Leone A. Enhanced visual spatial attention ipsilateral to rTMS-induced 'virtual lesions' of human parietal cortex. Nat Neurosci 2001;4: 953-957.

21. Snyder AW. Explaining and inducing savant skills: privileged access to lower level, less-processed information. Philos Trans R Soc Lond B Biol Sci 2009;364:1399-1405.

22. Gallate J, Chi R, Ellwood S, Snyder AW. Reducing false memories by magnetic pulse stimulation. Neurosci Lett 2009;449:151-154.

23. Oliveri M, Romero L, Papagno C. Left but not right temporal involvement in opaque idiom comprehension: a repetitive transcranial magnetic stimulation study. J Cogn Neurosci 2004;16:848-855.

24. Pascual-Leone A, Catalá MD. Lateralized effect of rapidrate transcranial magnetic stimulation of the prefrontal cortex on mood. Neurology 1996;46:499-502.

25. George MS, Nahas Z, Molloy M, et al. A controlled trial of daily left prefrontal cortex TMS for treating depression. Biol Psychiatry 2000;48:962-970.

26. Kim DR, Pesiridou A, O’Reardon JP. Transcranial magnetic stimulation in the treatment of psychiatric disorders. Curr Psychiatry Rep 2009;11:447-452.

27. Boggio PS, Rigonatti SP, Ribeiro RB. A randomized, double-blind clinical trial on the efficacy of cortical direct current stimulation for the treatment of major depression. Int J Neuropsychopharmacol 2008;11:249-254.

28. Nistche MA, Boggio PS, Fregni F, Pascual-Leone A. Treatment of depression with transcranial direct current stimulation (tDCS): a review. Exp Neurol 2009;219: 14-19.

29. Maeda F, Keenan JP, Pascual-Leone A. Interhemispheric asymmetry of motor cortical excitability in major depression as measured by transcranial magnetic stimulation. Br J Psychiatry 2000;177:169-173.

30. Mayberg HS. Frontal lobe dysfunction in secondary depression. J Neuropsychiatry Clin Neurosci 1994;6:428442.

31. Pascual-Leone A, Rubio B, Pallardó F, Catalá MD. Rapidrate transcranial magnetic stimulation of left dorsolateral prefrontal cortex in drug-resistant depression. Lancet 1996;348:233-237.

32. George MS, Wassermann EM, Williams WA. Changes in mood and hormone levels after rapid-rate transcranial magnetic stimulation (rTMS) of the prefrontal cortex. J Neuropsychiatry Clin Neurosci 1996;8:172-180.

33. Koenigs M, Ukueberuwa D, Campion P, Grafman J, Wassermann E. Bilateral frontal transcranial direct current stimulation: Failure to replicate classic findings in healthy subjects. Clin Neurophysiol 2009;120:80-84.

34. Nitsche MA, Fricke K, Henschke U, et al. Pharmacological modulation of cortical excitability shifts induced by transcranial direct current stimulation in humans. J Physiol 2003;553:293-301. 
35. Nitsche MA, Kuo MF, Karrasch R, Wächter B, Liebetanz $\mathrm{D}$, Paulus W. Serotonin affects transcranial direct currentinduced neuroplasticity in humans. Biol Psychiatry 2009; 66:503-508.

36. Young L, Camprodon JA, Hauser M, Pascual-Leone A, Saxe R. Disruption of the right temporoparietal junction with transcranial magnetic stimulation reduces the role of beliefs in moral judgements. Proc Natl Acad Sci USA 2010;107:6753-6758.

37. Knoch D, Pascual-Leone A, Meyer K, Treyer V, Fehr E. Diminishing reciprocal fairness by disrupting the right prefrontal cortex. Science 2006;314:829-832.

38. Fecteau S, Knoch D, Fregni F, Sultani N, Boggio P, PascualLeone A. Diminishing risk-taking behavior by modulating activity in the prefrontal cortex: a direct current stimulation study. J Neurosci 2007;27:12500-12505.

39. Lo YL, Fook-Chong S, Tan EK. Increased cortical excitability in human deception. Neuroreport 2003;14:10211024.

40. Priori A, Mameli F, Caogiamanian F, et al. Lie-specific involvement of dorsolateral prefrontal cortex in deception. Cereb Cortex 2008; 18:451-455.

41. Luber B, Fisher C, Appelbaum PS, Ploesser M, Lisanby $\mathrm{SH}$. Non-invasive brain stimulation in the detection of deception: scientific challenges and ethical consequences. Behav Sci Law 2009;27:191-208.

42. Buchanan A. Enhancement and the ethics of development. Kennedy Inst Ethics J 2008;18:1-34.

43. Wassermann EM. Risk and safety of repetitive transcranial magnetic stimulation: report and suggested guidelines from the International Workshop on the Safety of Repetitive Transcranial Magnetic Stimulation, June 5-7, 1996. Electroencephalogr Clin Neurophysiol 1998;108:1-16.

44. Been G, Ngo TT, Miller SM, Fitzgeral PD. The use of tDCS and CVS as methods of non-invasive brain stimulation. Brain Res Rev 2007;56:346-361.

45. Schonfeld T. Parents of unhappy poets: fiduciary responsibility and genetic enhancements. Camb Q Healthc Ethics 2003;12:411-417.
46. Keenan J, Gallup GG, Falk D. The Face in the Mirror: The Search for the Origins of Consciousness, 1st ed. New York: HarperCollins; 2003.

47. Riis J, Simmons J, Goodwin G. Preferences for enhancement pharmaceuticals: the reluctance to enhance fundamental traits. J Consum Res 2008;35:495-508.

48. Farah MJ, Illes J, Cook-Deegan R, et al. Neurocognitive enhancement: what can we do and what should we do? Nat Rev Neurosci 2004;5:421-425.

49. Sandel MJ. The case against perfection: what's wrong with designer children, bionic athletes, and genetic engineering. Atl Mon 2004;292:50-54, 56-60, 62.

50. Chatterjee A. "Cosmetic neurology" and the problem of pain. Cerebrum July 30, 2007.

51. Ochsner KN, Zaki J, Hanelin J, et al. Your pain or mine? Common and distinct neural systems supporting the perception of pain in self and other. Soc Cogn Affect Neurosci 2008;3:144-160.

52. Kramer PD. Listening to Prozac: The Landmark Book About Antidepressants and the Remaking of the Self, Revised Edition. New York: Penguin Group; 1997.

53. Conrad P. Medicalization of Society: On the Transformation of Human Condition into Treatable Disorders. Baltimore: Johns Hopkins University Press; 2007.

54. Scott CL, Holmberg T. Castration of sex offenders: prisoners' rights versus public safety. J Am Acad Psychiatry Law 2003;31:502-509.

55. McCabe SE, Knight JR, Teter CJ, Wechsler H. Nonmedical use of prescription stimulants among US college students: prevalence and correlates from a national survey. Addiction 2005;100:96-106

56. Sahakian B, Morein-Zamir S. Professor's little helper. Nature 2007;450:1157-1159.

57. Chatterjee A. Cosmetic neurology and cosmetic surgery: parallels, predictions and challenges. Camb Q Healthc Ethics 2007;16:129-137.

58. Illes J, Bird SJ. Neuroethics: a modern context for ethics in neuroscience. Trends Neurosci 2006;29:511-517. 